INVESTIGACIONES

\title{
PIZARRAS INTERACTIVAS PARA UN APRENDIZAJE MOTIVADO EN NIÑOS CON PARALISIS CEREBRAL
}

\author{
Enhancing motivated learning in cerebral palsied children through \\ interactive whiteboards
}

\author{
Ricardo Rosas, Claudia P. Pérez-Salas, Polín Olguín \\ Pontificia Universidad Católica de Chile, Escuela de Psicología, \\ Centro de Desarrollo de Tecnologías de Inclusión (CEDETI). \\ Fono: (056-02) 354 1520. E-mail: rrosas@uc.cl; cpperez@uc.cl
}

\section{Resumen}

Se presentan los resultados de un modelo de intervención, basado en la utilización de pizarras interactivas para promover la autoeficacia y la motivación en el aprendizaje de escolares con discapacidad motora severa que reciben educación especial. La intervención consistió en la realización de actividades educativas semanales a 32 alumnos (6-21 años), que a través de la integración de componentes de software y hardware permitieron trabajar los contenidos del currículum de una manera lúdica y colaborativa. Las actividades propuestas fueron filmadas y comparadas con las clases regulares. Los resultados indican un mayor grado de atención sostenida, mayor motivación y disfrute de las actividades en las clases interactivas. Asimismo, las entrevistas realizadas indican una evaluación positiva de la intervención respecto a la motivación, interacción y autoeficacia en el aprendizaje.

Palabras clave: pizarras interactivas, educación especial, discapacidad motora, aprendizaje colaborativo.

\begin{abstract}
This article shows the results of an intervention whose main objective was to enhance learning motivation and self efficacy in children with cerebral palsy attending to special education. The program included 32 students from 6 to 21 years old who participated in weekly educative sessions. These sessions incorporated learning activities using software and hardware supports that addressed their curriculum content in a playful and collaborative way. Each interactive session was recorded and compared with regular classes showing an increase in student's motivation and enjoyment of lessons. Moreover, children and teacher's interviews indicate a positive evaluation of the intervention results in terms of their impact in the group interaction, motivation and learning self efficacy.
\end{abstract}

Key words: interactive whiteboard, special education, motor disability, colaborative learning. 


\section{INTRODUCCION}

La enseñanza de niños con Parálisis Cerebral (PC) es un trabajo complejo y desafiante debido a las múltiples necesidades educativas especiales (NEE) que presentan estos menores. Tales NEE provocan que las estrategias instruccionales habituales no siempre sean apropiadas para ellos, dificultando el proceso enseñanza-aprendizaje. En este contexto, la búsqueda continua de nuevas estrategias educativas que permitan enseñar a los niños con PC de una manera más efectiva resulta extremadamente relevante, si se quiere desarrollar al máximo sus potencialidades.

Uno de los problemas que conllevan las rutinas regulares de enseñanza en estos niños es la dificultad para captar y mantener su atención. Esta dificultad no parece ocurrir cuando se enfrenta al niño a otro tipo de actividades como por ejemplo la televisión o la computación, sin embargo éstos no siempre pueden ser incorporados dentro de sus rutinas de aprendizaje en la escuela.

Capitalizar este interés a través de la incorporación de herramientas tecnológicas como la pizarra interactiva a la sala de clases puede ser una opción muy provechosa para los niños con PC, debido a los múltiples beneficios que ha demostrado su implementación en la enseñanza de niños preescolares, y escolares sin discapacidades y en la de niños y jóvenes con necesidades educativas especiales.

\section{APRENDIZAJE E INTERACCION}

Las tecnologías de la información y comunicación (TIC) se han convertido, durante las dos últimas décadas, en una parte importante del proceso enseñanza-aprendizaje en muchos establecimientos educacionales (Edwards, 2005). Pese a la reticencia inicial respecto a la pertinencia de utilizar medios informáticos en niños preescolares (3-5 años) hoy en día se reconoce que, incluso a tan temprana edad, la exposición a experiencias computacionales tienden a apoyar y favorecer el desarrollo cognitivo (en especial el aprendizaje verbal) y social de los alumnos (Goodwin, Goodwin \& Garel, 1996; Papert, 1980). De este modo, la investigación en los últimos 15 años ha enfatizado que la utilización de computadores en la enseñanza preescolar y escolar representa una experiencia de aprendizaje valiosa, especialmente cuando dicha tecnología es utilizada de manera pedagógica apropiada (Edwards, 2005).

En el caso de los niños con necesidades educativas especiales, las TIC como herramientas instruccionales también han demostrado ser útiles. Específicamente se ha encontrado que la enseñanza por medios interactivos con formas, sonidos y animación incrementa la motivación, atención y el tiempo que los alumnos con discapacidades invierten en las actividades; del mismo modo que favorece tanto las habilidades de prelectura como las de lectura (Boone, Higgins, Notari \& Stump, 1996; Williams, Wright, Callaghan, \& Coughlan, 2002).

Las TIC tienen la capacidad de aumentar la naturaleza cognitiva de las experiencias de aprendizaje y, en especial, la capacidad de los alumnos para comunicarse y expresarse (Jones, 2007). Algunos estudios han mostrado que muchos alumnos son altamente capaces para comunicarse con imágenes, colores, sonidos y música, pero que tienen dificultades para hacerlo con palabras, por lo que los recursos multimedia parecieran ser necesarios para estimular su pensamiento y creatividad, además de brindarles la posibilidad de rea- 
lizar múltiples intentos y recibir retroalimentación contingente por las tareas realizadas. Una educación focalizada en fomentar la literacidad únicamente a través de la escritura y lectura puede cortar el proceso necesario para desarrollar una comunicación exitosa. Lo anterior no implica negar la importancia de la escritura y lectura con palabras, sino manifestar que el enriquecimiento de las habilidades de literacidad en alumnos con discapacidad a través de actividades multimediales puede proveer una alternativa de éxito para aquéllos que les resulta difícil comunicarse tempranamente con medios tradicionales (Vincent \& Jones, 2007).

Hoy en día no parece haber dudas respecto a que los computadores efectivamente ayudan a los niños a aprender. Sin embargo, la discusión se ha trasladado ahora a cómo los recursos informáticos pueden ser utilizados para favorecer más eficazmente el aprendizaje, dado que no todas las formas de la tecnología pueden ser apropiadas para las necesidades particulares de distintas poblaciones (Cook, 2003). Edwards (2005) encontró que la integración exitosa de esta tecnología en la educación está influenciada por una variedad de factores que incluyen el nivel de conocimiento computacional del profesor, la selección apropiada del software con el que se trabajará, el acceso a tecnología actualizada y la ubicación de los computadores dentro de la sala.

El tipo de tecnología y la ubicación física dentro del aula parecen ser un aspecto trivial, sin embargo este hecho tiene gran influencia dentro del proceso de enseñanza. Aunque se ha demostrado que los computadores pueden ser una herramienta pedagógica efectiva, lo cierto es que la instrucción en formato de laboratorio, la escasez de equipos y el tamaño de la pantalla dificultan la generación de dinámicas interpersonales (Judge, 2001). Al ser el aprendizaje una actividad inherentemente social, resulta fundamental tener la posibilidad de reforzar las propias creencias y pensamientos a través de las respuestas o preguntas que otras personas realizan a la clase (aspecto extremadamente relevante para los niños con necesidades educativas especiales), actividad que se ve fuertemente entorpecida cuando se trabaja individualmente con un computador (SMART Technology, 2006).

Una alternativa a la utilización de computadores personales lo constituye la pizarra interactiva; esta tecnología ha demostrado ser particularmente útil en la enseñanza de preescolares, puesto que permite a los niños escribir o dibujar directamente en la superficie con los dedos, imprimir la imagen y/o grabarla en el computador. Desde una perspectiva pedagógica, las pizarras interactivas facilitan el aprendizaje activo de los menores, propician el trabajo grupal colaborativo (por su gran tamaño) y permiten la reutilización del material y el posterior análisis de la tarea realizada (debido a la posibilidad de grabar) (Kennewell \& Morgan, 2003).

La utilización de la pizarra interactiva en niños con necesidades educativas especiales resulta sumamente provechosa. En efecto, esta tecnología sustentada por softwares apropiados parece ser útil para niños con diversos estilos de aprendizaje (auditivo, kinestésico, visual) e incluso para niños con déficits visuales, intelectuales o motores (SMART Technology, 2006). Los alumnos con discapacidad visual han demostrado beneficiarse de la utilización de pizarras interactivas dentro de su clase, específicamente debido a su gran tamaño, ya que los menores que usualmente no son capaces de ver las imágenes en una pizarra son capaces de visualizarlas e interactuar con ellas (Cooper \& Clark, 2003 citado en SMART Technology, 2006). En el caso de los alumnos con discapacidad intelectual, la pizarra interactiva ha demostrado ser un medio efectivo de enseñanza para la lectura 
individual de palabras objetivo y el aprendizaje observacional de éstas cuando la lección se dicta grupalmente (Mechling, Gast \& Krupa, 2007).

Los alumnos con discapacidad motórica, a su vez, parecen beneficiarse de la instrucción mediante la pizarra interactiva debido a que los ayuda a focalizar su atención en la lección (debido al gran tamaño) y a interactuar con el material desplegado en la pizarra a través de movimientos gruesos sobre la pantalla (Wood, 2001). Del mismo modo, se han reportado experiencias exitosas de integración de esta tecnología en la enseñanza de alumnos con problemas de memoria debido a la utilización de letras con fondo de color y el mayor énfasis fonético que permiten las actividades realizadas en la pantalla interactiva (Salintri, Smith \& Clovis, 2002 citado en SMART Technology, 2006).

\section{OBJETIVOS}

Objetivos generales:

1. Fomentar el aprendizaje de los alumnos por medio del uso de actividades lúdicas en TIC interactivas.

2. Transferir las metodologías propuestas a los profesores de aula.

\section{Objetivos específicos:}

1. Aumentar la participación de los alumnos en clases a través de la pizarra interactiva.

2. Aumentar la motivación de los alumnos en clases a través de la pizarra interactiva.

3. Aumentar la atención de los alumnos en clases a través de la pizarra interactiva.

4. Aumentar la autoeficacia de los alumnos en clases a través de la pizarra interactiva.

5. Propiciar en los profesores la utilización de diferentes elementos multimedia en la pizarra interactiva.

6. Aumentar la autoeficacia de los profesores en el diseño de clases con pizarra interactiva.

\section{METODOLOGIA}

La intervención tuvo una duración de 4 meses y se realizó con un total de 32 alumnos entre 6 y 21 años y cuatro profesoras, pertenecientes a cuatro cursos de una escuela diferencial para trastorno motor de la Región Metropolitana.

La estructura de las sesiones y realización de las actividades de la intervención se basaron en un tipo de metodología activa y participativa. Fueron activas, porque los alumnos desarrollaron las actividades educativas de manera dinámica y variada, al aprender los contenidos del currículum a través de juegos y actividades multimedia; y participativas, porque los alumnos y las profesoras fueron los protagonistas centrales, tanto en el proceso de diseño de las actividades como durante su ejecución. 


\section{PROCEDIMIENTO}

1. CONVOCATORIA: La convocatoria estuvo a cargo de la directora del colegio, quien luego de recibir la propuesta del proyecto inicial realizó una selección de las profesoras que participarían en la intervención. La selección se efectuó teniendo en consideración que al menos la mitad de las profesoras tuviera conocimientos computacionales y de sus aplicaciones pedagógicas, de modo de que pudieran apoyar a quienes no contaran con tales conocimientos previos. Las otras dos profesoras fueron escogidas de acuerdo a su motivación con la participación en proyectos educativos, aun cuando no tuvieran conocimientos formales en la aplicación de la tecnología. Una vez definidas las docentes, se les invitó a participar y se les hizo entrega de los consentimientos informados para ser firmados por ellas y los apoderados de los alumnos de su curso.

2. PERIODO DE OBSERVACION: La intervención se inició con un período de observación a las profesoras y a los cursos participantes durante sus clases regulares de 45 minutos. La observación fue realizada una vez por semana, durante un mes, por dos psicólogas que serían las encargadas de ejecutar la intervención. El objetivo principal de esta etapa fue conocer: a) la dinámica general de cada clase y curso; b) los contenidos que se estaban abordando; c) las estrategias pedagógicas utilizadas por la profesora en el aula; d) el funcionamiento individual y grupal de los alumnos en las actividades; e) la motivación, el disfrute y la atención sostenida de los alumnos en las actividades realizadas.

El período observacional permitiría un primer acercamiento a los participantes y una mirada global de la dinámica de clases. Con ello se lograría planificar las actividades que serían utilizadas en las clases con pizarra interactiva y así adecuar los contenidos al nivel de cada curso.

3. INSTALACIONES: La pizarra interactiva fue instalada en el Centro de Recursos de Aprendizaje (CRA) del establecimiento.

Para que todos los alumnos pudieran hacer uso de la pizarra interactiva, se utilizaron diversos switches adaptados de acceso al computador y programas de barrido automático de la pantalla. Los programas de barrido permiten el movimiento automático del cursor por los distintos elementos presentes en la pantalla, mientras que los switches adaptados permiten emular el botón izquierdo del mouse y activar la zona que se desee. Se probaron diferentes tipos de switches y adaptaciones de acceso, así como distintas posiciones para la comodidad de los alumnos (ver Imagen 1).

4. CREACION DE LAS ACTIVIDADES: Las actividades fueron diseñadas combinando modalidades de trabajo individual y colectivo y se utilizaron reforzadores gráficos, auditivos y kinésicos. Las herramientas TIC contempladas fueron un PC, una pizarra interactiva SMART Board modelo 680 y actividades multimedia diseñadas ad hoc.

$\mathrm{Al}$ inicio de la intervención, las actividades fueron diseñadas por las psicólogas encargadas, las que se regían por un esquema básico que se desprendía de las observaciones en el aula y de las planificaciones entregadas por las profesoras respecto a contenidos y objetivos de la sesión (de acuerdo al currículo). La profesora de cada curso era la encargada de ejecutar la clase ante los alumnos con el material entregado. 


\section{Imagen 1}

Dispositivos de acceso al computador utilizados en la intervención

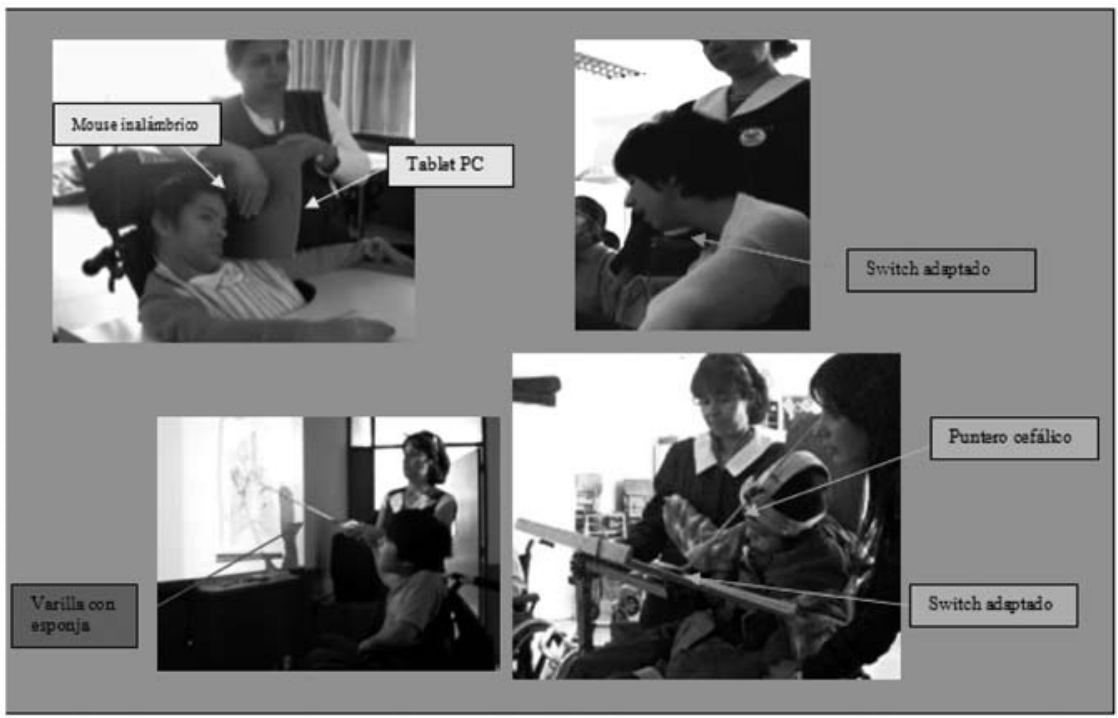

La estructura de cada sesión seguía los siguientes puntos:

a) Introducción: Al inicio de la clase se entregaban indicaciones de lo incluido en la actividad y de cómo realizarla, con reforzadores gráficos y auditivos.

b) Desarrollo: se desarrollaba la clase en base a las actividades educativas diseñadas en la pizarra de acuerdo a los diferentes contenidos propuestos.

c) Cierre: Se finalizaba la sesión apoyándose en reforzadores gráficos y auditivos para dar un cierre a los contenidos y felicitar a los participantes.

Luego de seguir este esquema por un mes, se solicitó a las profesoras que sugirieran actividades para ser diseñadas en la pizarra y luego se les entregaban convertidas en actividades multimediales. Un mes después de utilizar este procedimiento de diseño compartido, se les solicitó a las profesoras que idearan y diseñaran sus propias actividades multimedia de acuerdo a la estructura anterior.

5. COORDINACION: Se coordinó una primera reunión informativa con las profesoras y la directiva del colegio para explicar las actividades a realizar durante los cuatro meses que duraba la intervención con el uso de la pizarra interactiva. Para el procedimiento y creación de las actividades, se programaron reuniones semanales de 15 minutos entre la profesora y las psicólogas. Durante la transferencia de conocimiento a las profesoras para la creación de sus propias actividades, se fijó un horario semanal de 60 minutos para trabajar como apoyo en la elaboración de las clases. Este espacio permitiría a las profesoras desarrollar sus habilidades en el manejo de la pizarra interactiva y en su software. 
6. DESARRollo DE LAS ClASES: Se implementaron alrededor de seis sesiones por cada curso participante. Al inicio de la intervención se estableció una hora pedagógica (45 minutos) para cada sesión. Sin embargo, debido a la necesidad de traslado (desplazamiento de sillas de rueda) y funcionamiento adecuado de las herramientas tecnológicas, este horario se extendió a 90 minutos por sesión, lo que solucionó las dificultades que se presentaron.

La dinámica de clases con pizarra interactiva se desarrolló al igual que en una clase regular con la profesora jefe de cada curso participando como líder. La profesora guiaba cada sesión a través de indicaciones de la tarea a cumplir. De acuerdo a la actividad, la profesora otorgaba a los alumnos la posibilidad de trabajo individual o trabajo grupal donde todos pudieran participar.

La toma de turnos dependía de la actividad. En el trabajo individual, cada alumno tenía conciencia de su turno al aparecer su fotografía; en el trabajo grupal se hacía una ronda para que todos manejaran la pizarra interactiva.

7. TRANSFERENCIA: La transferencia de la responsabilidad en la creación de actividades fue un proceso gradual. Al inicio del proyecto, las psicólogas encargadas diseñaban las actividades multimedia en base a los contenidos que las profesoras definían. Posteriormente se les solicitó a las profesoras diseñar en papel las actividades de clase y las psicólogas las convertían a formato multimedia, enseñándoles el procedimiento. Finalmente, las profesoras debían diseñar y crear sus actividades multimedia bajo la supervisión de las encargadas, las cuales revisaban las rutinas y corregían posibles errores en el software.

8. DEVOLUCION DE LA INFORMACION: Una vez terminada la intervención se realizó una convivencia con los alumnos y profesoras participantes, además de los directivos del colegio, donde se les dio a conocer los principales resultados de la intervención y se les agradeció su colaboración.

\section{PLAN DE EVALUACION}

Para verificar el cumplimiento de los objetivos, se filmaron cuatro clases regulares (una por cada profesora) de 45 minutos cada una. Luego, al finalizar la intervención, es decir, luego de tres meses de experiencia con la nueva tecnología, se filmó nuevamente una clase por cada profesora, esta vez dictada con la utilización de la pizarra interactiva.

Para sistematizar la información obtenida de los videos respecto al funcionamiento grupal de los alumnos y de las profesoras se confeccionó una pauta global de evaluación, la que consideró los siguientes aspectos:

a) Atención sostenida: apreciación respecto al grado de atención que el alumno está prestando a la actividad, inferida a través de sus miradas, comentarios, entre otros aspectos.

b) Motivación: grado en que cada alumno demuestra compromiso con la actividad, entendida como disposición a repetir acciones, perseverar en la tarea, tratar de resolver dificultades, o iniciar por sí mismo actividades. 
c) Disfrute: grado en que cada alumno demuestra estar disfrutando la actividad, entendida como disposición a sonreír, verbalizar o demostrar agrado comportamentalmente (gritos o ruidos en el caso de niños sin vocalización).

Una segunda fuente de datos para la evaluación de la intervención lo constituyó la retroalimentación de los propios participantes. Para esto, se realizaron entrevistas individuales en profundidad a tres profesoras y a cuatro alumnos. Todas las profesoras fueron invitadas para ser entrevistadas, pero una de ellas no pudo asistir por dificultades de horario. Los alumnos fueron escogidos por conveniencia, de acuerdo a sus capacidades para expresarse de manera verbal.

Las entrevistas a los alumnos tuvieron una duración aproximada de 15 minutos cada una, mientras que las de las profesoras una duración aproximada de 30 minutos. Todas las entrevistas fueron filmadas, previa autorización verbal de los alumnos y escrita de los profesores. Los temas a indagar en las entrevistas se definieron de acuerdo a una pauta semiestructurada y se refirieron principalmente a las ventajas y desventajas de la utilización de la pizarra interactiva para el aprendizaje de los niños.

Los datos emanados de las entrevistas fueron codificados y categorizados en función de los temas comunes emergentes, y finalmente triangulados y complementados con los obtenidos de las filmaciones.

\section{ESTRUCTURA DE LAS SESIONES}

A continuación, se detallarán dos ejemplos de actividades que fueron implementadas durante la intervención. Las actividades prototipo son:

$\checkmark$ Actividad 1: Equivalencias de dinero

$\checkmark$ Actividad 2: Lectura funcional a partir de las señales del tránsito

1) ACTIVIDAD 1: EQUIVALENCIAS DE DINERO

- Visualización en la pantalla interactiva de una de las páginas de la actividad

- Descripción

La actividad se desarrolla en torno a la aplicación de equivalencias con billetes y monedas chilenas. El inicio de la actividad permite repasar los valores de monedas y billetes para posteriormente resolver algunos problemas simples. Los ejercicios consisten en juntar un valor que se consigna en la pizarra, para el cual existen diferentes alternativas: sólo billetes, billetes y monedas o sólo monedas.

La actividad puede ser llevada a cabo de dos maneras:

a) arrastrando las fotos de billetes o monedas a través de la pizarra hasta formar el monto solicitado.

b) presionando debajo de cada moneda (o el switch adaptado) para que caiga la moneda hasta la base de la pizarra. 
Imagen 2

Actividad 1 de equivalencias de dinero

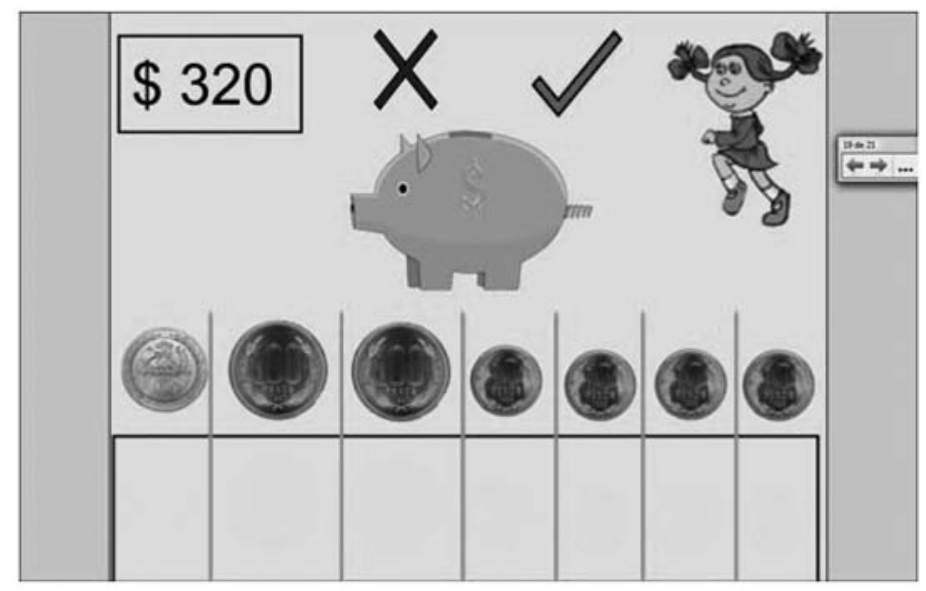

Cada vez que cae una moneda se escucha el sonido qué esta hace al caer en una alcancía.

Cuando el alumno ha formado correctamente el monto de dinero solicitado, la profesora presiona el símbolo de "visto bueno" y suenan aplausos. Cuando la respuesta es incorrecta suena una trompeta que indica que la alternativa es errónea.

\section{- Objetivo}

Conocer el valor de distintas monedas y billetes nacionales.

Conocer la equivalencia entre monedas y billetes.

\section{- Procedimiento}

En esta actividad todos los alumnos participan de manera individual respetando su turno, pero al mismo tiempo los compañeros pueden ayudar de forma colaborativa al compañero que se encuentre desarrollando la actividad. Se fomenta el trabajo colaborativo a pesar de trabajar individualmente. En el caso de los alumnos tetraparésicos, se utiliza el programa de barrido automático de la pantalla para que éstos puedan seleccionar autónomamente la alternativa que resuelve el problema.

\section{- Materiales}

Pizarra interactiva, switches adaptados, tablet PC y Vara de esponja.

Los programas utilizados son: Software Notebook 10, Audacity (sonido) y Kanghooru (barrido). 


\section{**Links Programas}

http://www2.smarttech.com/st/en-US/Support/Downloads/SBS/NBSv10WinEval.htm http://audacity.sourceforge.net/ http://www.xtec.cat/ jlagares/indexcastella.htm

2) ACTIVIDAD 2: LECTURA FUNCIONAL A PARTIR DE LAS SEÑALES DEL TRANSITO

- Visualización en la pantalla interactiva de una de las páginas de la actividad

\section{Imagen 3}

Actividad 2 de lectura funcional

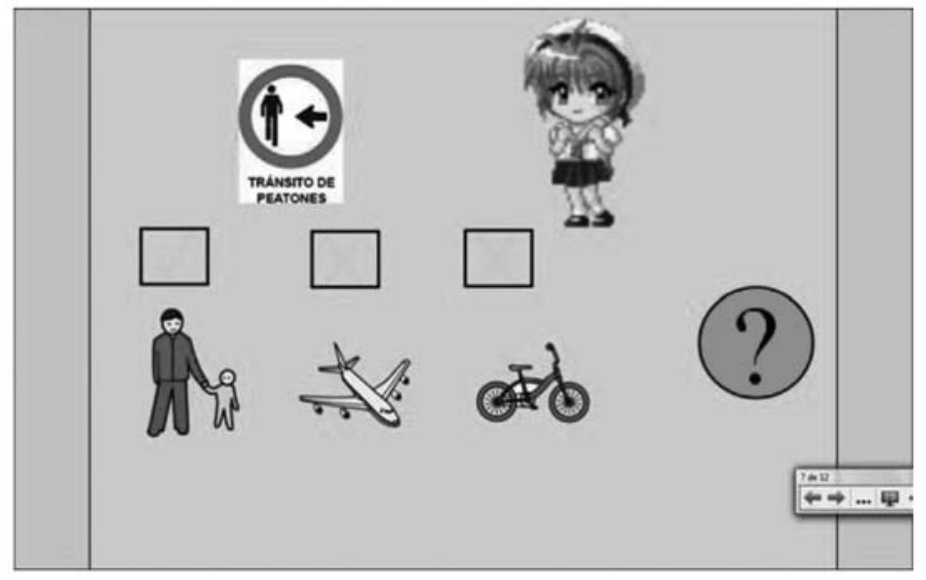

\section{- Descripción}

La actividad está enfocada al trabajo con las Señales del Tránsito. A partir de esto se trabajan actividades de diversa dificultad tales como ordenar oraciones, completar palabras, lectura y comprensión de textos cortos, términos pareados y también identificación de nombres. Para lograr estas tareas se presentan diversos estímulos fotográficos e icónicos que deben ser identificados, arrastrados o seleccionados para dar una respuesta. En los términos pareados, los alumnos pueden rayar con sus dedos o con un lápiz digital para resolver el problema; para ordenar las oraciones pueden usar sus manos para arrastrar las palabras y organizar con sentido la oración al ritmo de la música.

\section{- Objetivo}

Ejercitar el proceso de la lectura a nivel funcional con las Señales del Tránsito. 


\section{- Procedimiento}

Se diseñaron páginas adaptadas para cada nivel de aprendizaje. En este caso, se encontraban trabajando paralelamente cuatro niveles de aprendizaje que pudieron ser integrados a través de esta metodología. Se realizaron turnos que posibilitaron el desarrollo individual de cada alumno, permitiendo el espacio al trabajo grupal para solucionar las dificultades. Para que todos puedan resolver estos ejercicios, se utiliza el programa de barrido en la actividad para que el alumno decida cuándo hacer click y pueda resolver el problema que se le presenta.

\section{- Materiales}

Pizarra interactiva, switches adaptados, tablet PC y vara de esponja.

Los programas utilizados son: Software Notebook 10, Audacity (sonido) y Kanghooru (barrido).

\section{**Links Programas}

http://www2.smarttech.com/st/en-US/Support/Downloads/SBS/NBSv10WinEval.htm http://audacity.sourceforge.net/

http://www.xtec.cat/ jlagares/indexcastella.htm

\section{ANALISIS DEL PROCESO}

El presente análisis tiene por finalidad dar a conocer los resultados provenientes de dos fuentes de evaluación de esta intervención: a) la comparación de las filmaciones realizadas a clases regulares versus las realizadas a clases con pizarra interactiva y b) las opiniones de los participantes entregadas durante las entrevistas individuales al finalizar el proceso.

\section{A) COMPARACION DE FILMACIONES A CLASES REGULARES VERSUS CLASES CON PIZARRA}

El análisis de las filmaciones de clases regulares y clases con pizarra interactiva indica que, en términos generales, existe un cambio en la dinámica global de la clase. Las clases regulares son de ritmo lento, con actividades individuales donde la profesora se focaliza en un alumno con una actividad acorde a su nivel de aprendizaje, mientras los demás alumnos esperan su turno. Así, la clase de desarrolla de manera pausada, donde cada alumno desarrolla una actividad por una fracción limitada de tiempo y debe esperar varios minutos hasta que le toque nuevamente su turno. Las clases con pizarra interactiva, en cambio, tienen un ritmo rápido, la profesora se dirige al curso como una totalidad, y si bien existen actividades específicas para cada nivel de aprendizaje y cada alumno tiene su turno para responder, todos los demás compañeros pueden ver su respuesta y la corrección de ésta, por lo que las actividades tienen continuidad y dinamismo.

Otro aspecto observado en las clases regulares es la limitada focalización de la atención por parte de los alumnos. Al desarrollarse las actividades mayoritariamente de manera individual la atención y concentración se limita al desarrollo de la actividad 
propia y durante el tiempo en que la profesora está guiando su ejecución, pero mientras la actividad está siendo desarrollada por el compañero, los alumnos dejan de atender y su concentración en el trabajo propio también decae. Lo anterior se evidencia en que la mayoría de los alumnos que esperan su turno no dirigen la mirada hacia la actividad desarrollada por el compañero, y quienes lo hacen, desde el ángulo en que se encuentran, no pueden observar la respuesta de éste. En consecuencia, los alumnos dirigen su mirada a otras partes de la sala o bien interactúan entre ellos en otros asuntos, limitando incluso la atención hacia la propia actividad educativa a desarrollar.

En las clases con pizarra interactiva, en cambio, se aprecia un mayor grado de atención en las actividades propuestas. Esto se ve reflejado en la actitud corporal que presentan la mayoría de los alumnos durante el desarrollo de las clases, con su mirada atenta hacia la pizarra y hacia los dispositivos de acceso adaptados para la interacción con ésta. La mirada, los comentarios y/o vocalizaciones son congruentes con lo que se está desarrollando en la pizarra, lo que demuestra interés en la actividad que están ejecutando individualmente. Estos comportamientos también ocurren cuando la actividad está siendo desarrollada por los compañeros. Esta mejora en la atención, en comparación a las clases regulares, es particularmente más notoria en el caso de los niños con tetraparesia que además poseen anartria (ausencia de habla). En ellos se observa una clara dirección de su mirada y comportamientos no verbales contingentes con la clase que dan cuenta de que están siguiendo plenamente las actividades desarrolladas.

La interacción grupal también se vio alterada en las clases con pizarra interactiva en comparación a las clases regulares. En estas últimas había escasa interacción entre los alumnos, ocasionalmente había intercambio verbal entre aquéllos que podían vocalizar, pero muy escasa comunicación con los compañeros con los cuadros motores más severos. Estos alumnos permanecían en su sector de la sala y durante la clase interactuaban casi únicamente con la profesora cuando le tocaba su turno (1 ó 2 veces durante la clase). En las clases con pizarra interactiva, en cambio, se observa una mayor interacción entre los alumnos y la profesora. Cuando el alumno que está respondiendo no sabe la respuesta correcta, los compañeros que observan lo ayudan, lo aplauden, ríen cuando es acertada o le corrigen o hacen señas (o ruidos) cuando es errónea. Los dispositivos de acceso adaptados para los alumnos con cuadros motores más severos ayudan a esta interacción, puesto que los alumnos indican a la profesora cuándo el compañero tetraparésico quiere contestar y todos pueden observar cuál es la respuesta que éste da.

Otro elemento que se desprende de los videos es la mayor motivación de los alumnos al trabajar con la pizarra interactiva en comparación a las clases regulares. En las clases con pizarra interactiva se observa un mayor grado de compromiso con la clase, para terminar su actividad, repetirla, compartirla con sus compañeros y aprovechar cada momento para acercarse a la pizarra y responder a su turno. En muchas ocasiones, a pesar de equivocarse, los alumnos perseveran en su tarea hasta lograr el resultado esperado y hacer las cosas bien. Incluso, cuando surgen situaciones problemáticas entre todos buscan soluciones para resolverlas, lo que conlleva finalmente a sortear la dificultad en beneficio del avance del grupo. La motivación se aprecia también en el esfuerzo físico que realizan para manipular la pizarra por sí solos. Al parecer, interactuar con la pizarra, mover un objeto o presionar un switch por sí mismos, aun cuando les exige mucho esfuerzo, es una actividad que desean realizar, por lo que no cesan hasta conseguirla. Se aprecia también, que aunque se les ofrece ayuda para ejecutar estas labores, la mayoría de los 
alumnos no desea ser ayudado y muestran gran satisfacción cuando logran realizar las actividades de manera autónoma. En las clases regulares, no se aprecia, sin embargo, tan alta motivación. Los alumnos se esfuerzan cuando la profesora los incentiva, pero no se aprecia una motivación intrínseca por realizarlas.

Finalmente, respecto al disfrute de las actividades, se puede apreciar que en las clases con pizarra los alumnos parecen entretenerse y divertirse más que en las clases regulares. En las clases con pizarra interactiva se observan risas, la participación constante de los alumnos, incluso ansiedad por salir a la pizarra y resolver actividades. En otros casos, cuando los alumnos observaban el turno de otros compañeros, lo alentaban a seguir o ellos mismos intentaban responder la actividad.

En síntesis, es posible señalar que en comparación a las clases regulares, en las clases con pizarra interactiva se observa un aumento significativo en la motivación, atención, interacción y entretención de los alumnos.

\section{B) OPINIONES DE LOS PARTICIPANTES RESPECTO A LA INTERVENCION}

\section{1) Aspectos positivos de la intervención con pizarra interactiva}

Esta categoría reúne aquellas opiniones de los participantes respecto a las utilidades de la intervención educativa con pizarra interactiva. Tales beneficios se refieren principalmente a efectos en la dinámica de la clase, efectos en la dinámica grupal del curso y efectos en los alumnos de manera individual.

i) Efecto en la dinámica de la clase:

Los alumnos participantes señalan que la intervención con pizarra interactiva hace que la clase sea más entretenida, más divertida e interesante que las clases regulares. Asimismo, señalan que las actividades multimedia hacen más fácil el aprendizaje.

Lo anterior se puede apreciar en las siguientes citas:

“(con pizarra interactiva) se pueden hacer clases interesantes (...) se puede dibujar (...) nos reímos, hicimos todas esas cosas (...) y aprendimos también”. (Alumno 2, p. 2).

“en la sala estamos más aburridos, y acá con la pizarra (...) acá es distinto poh, acá es más divertido (...)”. (Alumno 4, p. 2).

La apreciación de los alumnos es compartida por las profesoras, quienes resaltan la versatilidad que permite la utilización de pizarras interactivas en la sala de clase, específicamente la posibilidad de diseñar actividades más apropiadas y personalizadas para cada niño, independientemente de la severidad de su trastorno motor o cognitivo.

En este sentido, una profesora señala:

“(...) rompes la barrera de la dificultad motora primero, rompes la barrera del alcance en el espacio... aun cuando ocupen la media pizarra hacia abajo, ya les agranda el espacio, el alcance de movimiento (...) Tienes ahí integrado quizás una sesión de terapia ocupacional, una sesión de kinesiología (...) a parte del contenido que tú puedes trabajar en la misma pizarra (...) (Profesora 1, p. 3). 
De la cita anterior se desprende el carácter más dinámico de las clases con pizarra interactiva, el cual está dado por la creatividad con la que se use el recurso y las actividades multimedia que se diseñen en él. De este modo, las profesoras señalan que dependiendo del objetivo que se propongan, las actividades pueden servir a propósitos muy diversos: desde desarrollar habilidades motoras hasta practicar habilidades cognitivas; y éstas pueden ser adecuadas según las características de cada alumno.

ii) Efecto en la dinámica grupal:

Esta subcategoría reúne aquellas percepciones de las profesoras y alumnos respecto al efecto que tuvo la intervención con pizarra interactiva en la dinámica grupal. En particular, las profesoras señalan que este recurso metodológico genera que el grupo se cohesione y se apoye mutuamente, fomentando la interacción y comunicación entre los compañeros.

Lo anterior se ve ejemplificado en la siguiente cita:

"yo creo que efectivamente el uso de la pantalla hace que el grupo se cohesione, hace que el grupo se apoye. Están todos pendientes de lo que el compañero está haciendo, algunos ya saben la respuesta, hasta se anticipan en los aplausos y en el 'cua cua cua' (sonido de error), lo que no sucede creo yo con un trabajo que uno hace en una sala, donde cada uno de ellos en el fondo es un mundito que está trabajando aparte. Creo que con esto (la intervención) se logra, que si bien es cierto cada uno está trabajando solo, también están en un macro, trabajando juntos, se apoyan y se motivan (...). (Profesora 3, p. 4).

Este efecto en la dinámica grupal no se aprecia explícitamente en el discurso de los alumnos entrevistados, pero puede verse de manera implícita en comentarios tales como el siguiente:

"Me gustaba más (las clases con pizarra interactiva) porque a veces lo aplaudíamos a "Manuelito" (...) a veces a mí (...)”. (Alumno 1; p. 3-4).

En la cita anterior se observa que el niño reconoce el valor de la interacción del grupo en comparación a las clases regulares.

iii) Efecto en cada alumno:

Esta subcategoría contempla las impresiones de los participantes respecto al efecto que la intervención con pizarra interactiva tuvo en ellos. Los alumnos destacan principalmente los efectos de la intervención en su estado de ánimo durante la clase, así como efectos en su sentido de autoeficacia y en el aprendizaje.

Lo anterior se ve ejemplificado en las siguientes citas:

"Me pareció bien (la intervención) (...) lo hice bien y lo trabajé solito (...) igual me costó, pero me gustó (...) con pizarra aprendo más, sí, y lo hago muy bien”. (Alumno 1, p. 1-2).

"me sale más fácil aprender, porque las cosas que yo no las entiendo y las veo ahí en la pizarra y me sale más fácil aprender". (Alumno 4, p. 3). 
“(En las clases con pizarra interactiva estábamos) contentos, felices, aprendí (...) La tía hacía actividades y todos la hacían bien (...) Ayuda a que aprendamos más cosas, que sepamos más cosas, que trabajemos más ahí, que le pongamos más empeño”. (Alumno 3, p. 2-3).

De las anteriores citas se desprende que los alumnos entrevistados señalan haber estado contentos y felices durante las clases con pizarra interactiva, además de sentirse orgullosos cuando podían resolver las actividades por sí solos. Destacan también el efecto positivo en el aprendizaje.

Las profesoras, por su parte, destacan los efectos de la intervención con pizarra interactiva en la motivación de los alumnos y en su autoestima. También mencionan los efectos positivos de la intervención sobre la concentración y atención de los alumnos en comparación a las clases regulares. Esto contribuiría, en opinión de las entrevistadas, a un mejor aprendizaje.

Lo anterior se evidencia en las siguientes citas:

“(la pizarra) es un recurso metodológico muy motivador para los chiquillos (...) eso es lo importante para ellos, poder hacer cosas con sus manos, subirles la autoestima (...) se pueden hacer cosas que a lo mejor ellos nunca pensaron que podían hacer, y desde ese punto de vista es muy importante". (Profesora 2, p. 2).

"La motivación que produce una pizarra interactiva, con los colores, con lo que es multimedia, a los chiquillos le aumenta la atención, la concentración (...) En relación a los aprendizajes, específicamente en el área en que yo la utilicé (lenguaje y comunicación) yo diría que avanzaron un $100 \%$ (...) Objetivamente vimos que ellos ordenaron oraciones en forma correcta, abordaron lectura global, que asociaron una palabra con lectura total o global a un significado (...) estoy muy contenta". (Profesora 1, p. 3).

Del discurso de las profesoras se desprende que reconocen el alto poder motivador de las actividades diseñadas en la pizarra interactiva, además del sentido de autoeficacia que les generó a sus alumnos ser capaz de manipularla y emitir respuestas de manera autónoma. Esto, en opinión de las entrevistadas habría potenciado el aprendizaje de los alumnos al generar un mayor esfuerzo, atención y concentración en las actividades.

\section{2) Aspectos a mejorar en la intervención con pizarra interactiva}

En esta segunda categoría se exponen las opiniones de los entrevistados en relación con los aspectos susceptibles de ser mejorados en la intervención. Destacan temas de carácter técnico y aspectos de implementación que interfirieron en el óptimo desarrollo de las actividades.

\section{i) Aspectos técnicos:}

La presente subcategoría detalla los elementos que, en opinión de las profesoras entrevistadas, dificultaron el trabajo durante el proceso. Aparece como principal obstaculizador la altura de la pizarra, la que debido a su ubicación, medio metro sobre el suelo, permitía que los niños utilizaran sólo la mitad inferior de ésta (debido a que la mayoría de los alumnos estaban sentados en sillas de ruedas). Asimismo, el hecho de tener que trasladarse desde las salas regulares a la sala donde se ubicaba la pizarra interactiva es 
visto como un problema debido a que en ese lugar no se encuentran todos los implementos que los alumnos necesitan para ejecutar las actividades (mesas adaptadas, sujetadores que los mantienen en posición corporal correcta, entre otros).

Lo anterior se ve graficado en las siguientes citas:

“(...) allá (sala regular) tenemos todos los recursos, tenemos las mesas adecuadas, si nos falta algún material como para implementar en el minuto en la actividad que se necesita para algún niño, uno va y sabe donde lo tiene, va y lo toma. Acá eso uno no lo podía prever, y a veces surgen estos imprevistos y no teníamos el material en el minuto". (Profesora 2, p. 4).

“(...) la altura de la pantalla, yo creo que habría que regularla (...). Pero efectivamente eso les complica a los chicos que no pueden acceder a la pantalla por las sillas (...)”. (Profesora 3 , p. 2).

Los alumnos entrevistados concuerdan en considerar un obstaculizador la ausencia de sus implementos habituales, debido a la incomodidad que les generaría. La altura de la pizarra no fue mencionada por los alumnos como una dificultad.

La siguiente cita refleja la opinión de los alumnos entrevistados:

"En la sala, el otro día, dijeron (los compañeros) que les gustaba venir, pero era muy incómodo moverse pa' allá y pa' acá... Es más fácil trabajar en las clases normales porque uno está acomodado en la mesa. (Alumno 2, p. 1).

Respecto al diseño de las actividades propiamente tal, se menciona que éstas mejorarían si se eliminaran las figuras con animación flash, debido que en opinión de las profesoras entrevistadas, éstas distraerían a algunos alumnos. Lo anterior se ve ejemplificado en la siguiente cita:

"Quizás bajaría un poco los distractores, en el sentido de los elementos que hablan y se mueven, dependiendo de los alumnos (...). Hay algunos niños que se distraen mucho con estos elementos de presentación". (Profesora 1, p. 8).

Algunos alumnos comparten esta opinión y otros señalan que las actividades les gustaron tal cual eran. Tal como es posible apreciar en el siguiente extracto:

“(...) igual nos entretuvimos harto, pero yo encontraba que algunas veces los niños nos distraíamos(...) porque esa niña, esa niña que saltaba, que corría, la niña que aparecía allí en la pantalla cuando hacíamos la actividad de matemáticas". (Alumno 3, p. 1).

"Me gustó trabajar con las monedas, después trabajar el sistema respiratorio, circulatorio (...) me gustó muchísimo (...) los sonidos (...)”. (Alumno 1, p. 1).

De las entrevistas realizadas se desprende que para algunos niños las animaciones flash pueden resultar distractores, especialmente aquéllos con problemas de discriminación visual. Sin embargo, para otros niños estos elementos pueden resultar motivantes y entretenidos.

ii) Implementación:

La subcategoría implementación se refiere a aquellos factores de implementación de la intervención que dificultaron el óptimo desarrollo de las actividades. En especial, se 
destacan las dificultades de coordinación entre las profesoras participantes del estudio y el poco tiempo y espacio para compartir, dentro de la jornada laboral, tanto las dificultades como los aciertos que se iban teniendo en el transcurso de la intervención.

Tales impresiones pueden observarse en las siguientes citas:

"Mira, yo creo que por un lado falta tiempo acá en el colegio, porque nunca tuvimos un tiempo destinado a preparar actividades para pantalla, entonces creo que ese fue uno de los grandes peros. Tampoco pudimos reunirnos con las otras chiquillas, por un tema de horario por tema de tiempo dentro del colegio (...) En ese sentido, pero creo que hubiera sido muy enriquecedor haber tenido una instancia de poder habernos reunido con las chiquillas una vez a la semana (...)”. (Profesora 3, pp. 1-2).

En este sentido, las profesoras destacan como principal obstaculizador para el óptimo funcionamiento de la intervención el escaso tiempo que poseen los profesores dentro de su jornada laboral para poder diseñar actividades en una pizarra interactiva. Si bien se reconoce que una vez realizadas, las actividades pueden reutilizarse como plantillas, al inicio del trabajo requiere mucho tiempo y dedicación. Asimismo, en el caso de la intervención, señalan que este trabajo podría haber sido más llevadero si hubiese existido la instancia de poder compartir con las demás profesoras participantes aprendizajes, datos o información para el desarrollo de las actividades.

\section{DISCUSIONES Y CONCLUSIONES}

La intervención "pizarras interactivas para un aprendizaje motivado" tuvo un efecto positivo en la motivación, interacción, atención sostenida y disfrute de las actividades por parte de los alumnos con discapacidad motora participantes en el estudio. Tales resultados son concordantes con lo que señala la teoría, respecto a que las TICs, como medio de aprendizaje, incrementan la motivación, la atención y disminuyen el tiempo invertido en la realización de las tareas (Edwards, 2005).

El aporte de la intervención con pizarra interactiva en el ámbito de la discapacidad motora expande los resultados obtenidos en la educación regular al permitir atender, mediante un diseño de actividades individualizadas, distintos tipos de discapacidades concomitantes que son frecuentes en los alumnos con trastorno motor (visuales, cognitivas, auditivas, entre otras). La versatilidad de las actividades diseñadas y los dispositivos de acceso adaptados al computador permiten, además, disminuir la barrera del movimiento para potenciar el aprendizaje en todos los alumnos.

Un elemento que emergió como obstaculizador dentro del proceso, y que además es un factor reconocido en otros estudios (e.g., Edwards, 2005; Judge, 2001), es la ubicación física de la herramienta tecnológica utilizada, que en este caso fue la pizarra interactiva. La ubicación física de la pantalla, medio metro sobre el suelo, impidió su óptimo funcionamiento dado que los alumnos sólo alcanzaban a tocar la mitad inferior de la pizarra. Asimismo, la instalación de la pantalla en el centro de recursos del aprendizaje del establecimiento fue un factor que interfirió en el correcto funcionamiento de la intervención, puesto que fue necesario suspender varias sesiones debido a que el colegio requería utilizar la sala para realizar reuniones de apoderados, consejos de profesores, entre otros, lo que afectó la continuidad de las clases planificadas. Lo anterior pone en evidencia, que el 
espacio físico y la ubicación específica de la pantalla en el establecimiento educacional es un hecho importante a la hora de proponer una intervención de este tipo.

Un segundo elemento que aparece como obstaculizador, lo constituye el escaso tiempo que los docentes poseen para diseñar actividades en la pizarra interactiva, reunirse con los encargados del proyecto y las demás profesoras participantes para compartir experiencias. De lo anterior se desprende la necesidad de incorporar a la intervención propuesta un período de capacitación docente paralelo a la intervención, de modo que el tiempo destinado a ésta esté contemplado y garantizado en su jornada.

En este mismo sentido, la comunicación con las profesoras debe ser directa, es decir, un trabajo conjunto entre el equipo encargado del proyecto y las profesoras participantes, de modo de diseñar e implementar, sin intermediarios, las tareas a realizar. Asimismo, es recomendable para este tipo de intervenciones mantener continuamente reuniones colaborativas entre todos los participantes, ya que son instancias muy necesarias para el intercambio de experiencias, información y materiales.

Los elementos que permitieron un positivo desarrollo del proyecto están relacionados a las características de las pizarras interactivas, ya que éstas permiten la accesibilidad para todos, incluyendo a niños con PC severa sin mayores problemas. Esto se hizo posible gracias a la utilización de diferentes dispositivos adaptados de acceso al computador que permitieron que todos los alumnos pudieran participar de las actividades y emitir sus respuestas de manera autónoma.

También, las características propias de la pizarra facilitan la posibilidad del uso de diversos softwares educativos y de entretenimiento, lo que asemeja el funcionamiento de un computador tradicional, pero de manera interactiva. En este sentido, es preciso enfatizar que no es la pizarra interactiva por sí misma la que genera el impacto positivo en la enseñanza de los alumnos con discapacidad motora, sino todas las actividades interactivas que ésta permite diseñar. Lo anterior implica, que para lograr resultados positivos como los obtenidos se requiere un diseño planificado de actividades que consideren los objetivos de las profesoras y las necesidades particulares de cada niño para crear actividades claras, didácticas, interactivas y entretenidas.

Finalmente, es posible señalar que los resultados obtenidos en la intervención generaron en las profesoras participantes, y en los docentes del colegio en general, una valoración muy positiva de la pizarra interactiva y las actividades multimedia desarrolladas en ésta. La positiva evaluación de la experiencia por parte de los participantes, así como su incorporación y apropiación de los recursos tecnológicos al final del proceso hacen proyectar resultados favorables respecto a la trasferencia de lo aprendido a su quehacer cotidiano en clases.

\section{BIBLIOGRAFIA}

Boone, R., K. Higgins, A. Notari, \& C. S. Stump (1996). Hypermedia pre-reading lessons: Learner-centered software for kindergarten. Journal of Computing in Childhood. Education, 7, 39-69.

Cook, D. (2003). The Question is No Longer 'If', but 'How Best', ICT can be Used in Early Years Practice. En Proc. Young Children and Learning Technologies. Artículos seleccionados desde International Federation for Information Processing Working Group 3.5 Open Conference, 
Melbourne, Australia. CRPIT, 34. Wright, J., A. McDougall, J. Murnane, and J. Lowe, Eds. ACS, 31-38.

Edwards, S. (2005). Identifying the factors that influence computer use in early childhood classroom. Australian Journal of Educational Technology, 21 (2): 192-210.

Goodwin, L., W. Goodwin, \& M. Garel (1996). Use of microcomputers with preschoolers: A review of the literature. Early Childhood Research Quarterly, 1, 269-286.

Jones, A. (2007). ICT, language, media: essential literacies for today. Redesigning pedagogy: Voices of practitioners. Singapore: Pearson.

Judge, S. L. (2001). Computer applications in programs for young children with disabilities. Journal of Special Education Technology, 16, 29-40.

Kennewell, S. \& A. Morgan (2003). Student teachers' experiences and attitudes towards using interactive whiteboards in the teaching and learning of young children. En Young children and learning technologies, eds. Wright, J.; A. McDougall, J. Murnane and J. Lowe. Sydney: Australian Computer Society. 71-76, 2003.

Mechling, L. C., D. L. Gast \& K. Krupa (2007). Impact of smart board technology: an investigation of sight word reading and observational learning. J Autism Dev Disord, 37, 1869-1882.

Papert, S. (1980). Desafío a la mente. Computadoras y Educación. Buenos Aires: Ediciones Galápago.

SMART Technologies Inc. (2006, marzo). Interactive whiteboards and learning: Improving student learning outcomes and streamlining lesson planning. Recuperado julio 10, 2008, desde Smarttech: http://education.smarttech.com/NR/rdonlyres/6EC7C51B-FC2A-4F95-B04B-B24C193C870B/0/ IntWhiteboardResearchWhitepaper.pdf.

Vincent, J. \& A. Jones (2007). Early years students working with multimedia tools. ICT in Education, 30 (2): 4-7.

Williams, C., B. Wright, G. Callaghan \& B. Coughlan (2002). Do children with autism learn to read more readily by computer assisted instruction or traditional book methods? Autism, 6, $71-91$.

Wood, C. (2001). Interactive Whiteboards -a luxury too far? Teaching ICT, 2, 52-62. 
